\title{
Propiedades reológicas de quinua (Chenopodium quinoa Wild) obtenidas mediante molienda abrasiva y tratamiento térmico
}

\author{
María P. Polo, Diego F. Roa* y Jesús E. Bravo \\ Departamento de Ingeniería Agroindustrial, Universidad del Cauca, Popayán; Calle 5 No. 4 - 70, Popayán - 190003, \\ Colombia (correo-e: mariapolo@unicauca.edu.co; jebravo@unicauca.edu.co; *droa@unicauca.edu.co)
}

* Autor a quien debe ser dirigida la correspondencia.

Recibido Mar. 29, 2021; Aceptado May. 28, 2021; Versión final Jun. 30, 2021, Publicado Dic. 2021

\begin{abstract}
Resumen
El objetivo de esta investigación fue determinar el efecto de la molienda abrasiva y el tratamiento térmico sobre las propiedades reológicas de harinas de quinua. Se determinó que la molienda abrasiva era apropiada para obtener harinas del germen y del perisperma, pero el tratamiento térmico modificó las propiedades reológicas. El modelo de la potencia fue empleado para determinar el índice de flujo (n) y la consistencia (k). Los resultados evidenciaron diferencias significativas entre el índice $\mathrm{n}$ de las harinas obtenidas del germen y del perisperma. Se observó un comportamiento dilatante a bajas concentraciones y pseudoplástico a altas concentraciones. Las velocidades de corte mayores a $200 \mathrm{~s} \mathrm{~s}^{-1}$ provocaron errores en la determinación de comportamiento reológico. Se observó un comportamiento Newtoniano en las dispersiones sometidas al proceso de calentamiento/enfriamiento, provocando una viscosidad estable a velocidad de corte entre 0 a $200 \mathrm{~s}^{-1}$. Se concluye que el modelo de la potencia se ajusta satisfactoriamente, logrando describir el comportamiento reológico de harinas de quinua.
\end{abstract}

Palabras clave: índice flujo; consistencia; harina de quinua; procesamiento térmico

\section{Rheological properties of quinoa (Chenopodium quinoa Wild) flours obtained by abrasive milling and heat treatment}

\begin{abstract}
The main objective of this research study was to determine the effect of abrasive grinding and heat treatment on the rheological properties of quinoa flours. Preliminary experiments showed that abrasive milling is appropriate for obtaining germ and perisperm flours, while hydrothermal treatment modified rheological properties. The power model was used to determine flow index $(\mathrm{n})$ and consistency $(\mathrm{k})$. The results showed significant differences in the $\mathrm{n}$ index between germ and perisperm flours. A shear thickening behavior was observed at low concentrations and a pseudoplastic behavior at high concentrations. Shear rate greater than $200 \mathrm{~s}^{-1}$ caused errors in the determination of rheological behavior. A Newtonian behavior was observed in dispersions subjected to the heating/cooling process, resulting in a stable viscosity at shear rate between 0 to $200 \mathrm{~s}^{-1}$. It is concluded that the power model applied here was adequate since it successfully characterized the rheological behavior of quinoa flours.
\end{abstract}

Keywords: flow index; consistency; quinoa flour; thermal treatment 


\section{INTRODUCCIÓN}

En la actualidad los consumidores buscan alimentos con funciones biológicas que aporten nutrientes esenciales y beneficiosos para la salud que impacten de manera positiva en el organismo para las funciones vitales, siendo los cereales y pseudocereales estos tipos de alimentos (El-Sohaimy et al., 2018). En años recientes, se ha despertado un interés por el aprovechamiento integral de los granos (Gupta et al., 2021) lo que ha requerido la innovación en los métodos de molienda (Awika et al., 2005) y como resultado el fraccionamiento efectivo del grano (Roa et al., 2019). La quinua es un cultivo de origen andino nutricionalmente denso y contiene una cantidad considerable de proteínas (Pereira et al., 2019), carbohidratos, grasas (Song et al., 2021), vitaminas, minerales y fibra dietética (Asher et al., 2020; Gupta et al., 2021); es una fuente importante de proteínas debido a su digestibilidad y su composición equilibrada enaminoácidos esenciales (Dussán-Sarria et al., 2019). Morfológicamente el grano de quinua está formado por el epispermo, el embrión y el perispermo.

El proceso de molienda abrasiva se ha utilizado en granos de forma esférica para eliminar partes del pericarpio (Awika et al., 2005) siendo la molienda abrasiva un medio eficaz que permite la eliminación de saponinas por medio de descascarillado de la quinua, además permite obtener dos fracciones en el grano de quinua que son estructuralmente diferentes como lo es el germen y el grano pulido que no es otra cosa que el perisperma almidonoso (Roa et al., 2019). Por lo tanto, el uso de la molienda abrasiva permite obtener harinas con alto potencial tecnológico para el desarrollo de productos, y es por esta razón, que resulta necesario conocer las características fisicoquímicas, reológicas y estructurales de las harinas, esto con el fin de lograr un adecuado desarrollo de una formulación.

Actualmente se han aplicado procesos tecnológicos como las altas presiones sobre polvos alimenticios para mejorar propiedades como la solubilidad en alimentos reconstituidos, así como, en la estabilidad de la viscosidad en las dispersiones durante el proceso de pasterización (Warncke y Kulozik, 2020); pero este proceso de altas presiones algunas veces requiere equipamiento de alto costo, lo cual no es conveniente para pequeñas empresas, sin embargo, se pueden lograr buenos resultados aplicando tratamiento térmicos, los cuales pueden modificar las proteínas presentes en el alimento (Mulcahy et al., 2017), así como, provocar una gelatinización total o parcial del almidón (Roa et al., 2019); es así como, el tratamiento térmico puede modificar las propiedades reológicas y los atributos de calidad de las harinas (Romero y Zhang, 2019; Romano et al., 2020; Arzuaga et al., 2021).

Por lo anterior el tratamiento térmico se puede utilizar en harinas para obtener dispersiones de baja viscosidad, pero estables a los ciclos de calentamiento y enfriamiento, logrando la diversificación de usos de la harina como una opción tecnológica para desarrollar productos resistentes a los tratamientos térmicos. En este trabajo, se tiene presente el factor de innovación en el uso de harinas de quinua para la elaboración de productos funcionales con altos contenidos en proteína y para ello se realizan pruebas reológicas para determinar comportamientos de proceso que presenta la harina de quinua y a su vez identificar los efectos que podría presentar mediante tratamientos térmicos.

\section{METODOLOGÍA}

La metodología se presenta en subsecciones: en una se describe el grano de quinua, en la segunda se describen los procesos tecnológicos aplicados al grano de quinua y sus harinas y en la tercera subsección se detallan los métodos para determinar las propiedades reológicas.

\section{Materiales}

Se utilizaron granos provistos y adecuados por la empresa SEGALCO S.A.S.; la variedad fue Tunkahuan, el cual fue cosechado en el municipio de Bolívar-Cauca al sur de Colombia. Esta variedad de grano de quinua presenta un contenido de saponina de 0.06 y $15.73 \%$ de proteína aproximadamente. Sus principales componentes son almidón $64,2 \%$ a $70,4 \%$ de materia seca, proteínas $11,0 \%$ a $16,5 \%$, lípidos $4,1 \%$ a $7,5 \%$ y fibra dietética 3,8\% a 19,7\% (Delgado et al., 2009; Van de Vondel et al., 2021)

\section{Molienda Abrasiva}

Se empleo un Molino experimental arrocero (GranEL, model C-100, Bogotá, Colombia), el cual tiene una cámara de abrasión usada para la obtención de harinas (FP10 y FP40) y grano pulido (C10 y C40) a partir del grano entero de quinua. El grano pulido fue pasado por un molino analítico de cuchillas para la obtención de las harinas. Las harinas fueron pasadas por tamices de abertura de $250 \mu \mathrm{m}$ y $125 \mu \mathrm{m}$ para los respectivos análisis. 


\section{Tratamiento térmico}

Se prepararon dispersiones de harina en agua con una relación (1:3), posteriormente las dispersiones se homogenizaron durante 2 minutos a $9000 \mathrm{rpm}$ en un equipo ultraturrax, luego las dispersiones fueron calentadas a $90 \stackrel{\circ}{\circ}$ durante 40 minutos empleando el termostato marca polystat, posteriormente las muestras se secaron a $50 \stackrel{\circ}{ } \mathrm{C}$ durante 16 horas en un horno de convección forzada, y finalmente las harinas obtenidas se almacenaron a $5{ }^{\circ} \mathrm{C}$, en bolsas de polietileno para evitar transferencia de agua al material.

Todas las muestras utilizadas en este estudio se obtuvieron mediante molienda abrasiva del grano entero, obteniéndose una harina fina y un grano pulido, las cuales fueron sometidas a un proceso térmico; las condiciones experimentales se describen a continuación: la muestra C-10-125-6\% es una dispersión de harina del grano pulido al $6 \% \mathrm{p} / \mathrm{v}$ con una granulometría inferior a $125 \mu \mathrm{m}$ obtenida a los $10 \mathrm{~min}$ de la molienda. La muestra C-40-125$6 \%$ es una dispersión de harina del grano pulido al $6 \% \mathrm{p} / \mathrm{v}$ con una granulometría inferior a $125 \mu \mathrm{m}$ obtenida a los 40 min de la molienda. La muestra C-10-125-12\% es una dispersión de harina del grano pulido al $12 \% \mathrm{p} / \mathrm{v}$ con una granulometría inferior a $125 \mu \mathrm{m}$ obtenida a los $10 \mathrm{~min}$ de la molienda. La muestra C-40-125-12\% es una dispersión de harina del grano pulido al $12 \%$ p/v con una granulometría inferior a $125 \mu \mathrm{m}$ obtenida a los $40 \mathrm{~min}$ de la molienda. La muestra FP-10-125-6\% es una dispersión de harina del germen del grano al $6 \%$ p/v con una granulometría inferior a $125 \mu \mathrm{m}$ obtenida a los 10 min de la molienda. La muestra FP-40-125-6\% es una dispersión de harina del germen del grano al $6 \% \mathrm{p} / \mathrm{v}$ con una granulometría inferior a $125 \mu \mathrm{m}$ obtenida a los $40 \mathrm{~min}$ de la molienda. La muestra FP-10-125-T-6\% es una dispersión de harina del germen del grano al $6 \% \mathrm{p} / \mathrm{v}$ con una granulometría inferior a $125 \mu \mathrm{m}$ obtenida a los 10 min de la molienda y sometida a un tratamiento térmico previo. La muestra FP-40-125-T-6\% es una dispersión de harina del germen del grano al $6 \%$ p/v con una granulometría inferior a $125 \mu \mathrm{m}$ obtenida a los $40 \mathrm{~min}$ de la molienda y sometida a un tratamiento térmico previo. La muestra FP-10-250-T-6\% es una dispersión de harina del germen del grano al $6 \% \mathrm{p} / \mathrm{v}$ con una granulometría inferior a $250 \mu \mathrm{m}$ obtenida a los $10 \mathrm{~min}$ de la molienda y sometida a un tratamiento térmico previo. La muestra FP-40-250T-6\% es una dispersión de harina del germen del grano al $6 \%$ p/v con una granulometría inferior a $250 \mu \mathrm{m}$ obtenida a los 40 min de la molienda y sometida a un tratamiento térmico previo. La muestra C1-125-T-6\% es una dispersión de harina del grano entero al $6 \%$ p/v con una granulometría inferior a $125 \mu \mathrm{m}$ y sometida a un tratamiento térmico previo.

\section{Propiedades de flujo}

Las propiedades de flujo de cada dispersión se determinaron mediante un reómetro (TA INSTRUMENTS, AR 1500, New Castel, EE. UU.), equipado con una geometría de cilindro concéntrico. La velocidad de cizallamiento se incrementó de 0 a $300 \mathrm{~s}^{-1}$ durante 5 minutos. La viscosidad aparente media se determinó entre 180 y $200 \mathrm{~s}^{-1}$, evitando la zona de transición por efecto de la velocidad de corte. Se obtuvieron curvas de flujo (esfuerzo cortante versus velocidad de corte) y se ajustaron al modelo de ley de potencia Eq. (1)

$$
\mathrm{t}=\mathrm{K}^{*}(\mathrm{Y})^{\mathrm{n}}
$$

Donde t es el esfuerzo cortante $(\mathrm{Pa}), \mathrm{Y}$ es la velocidad de corte $\left(\mathrm{s}^{-1}\right), \mathrm{K}$ es el coeficiente de consistencia $\left(\mathrm{Pa} . \mathrm{s}^{-1}\right)$ y $\mathrm{n}$ es el índice de comportamiento del flujo. El índice de comportamiento de flujo indica un comportamiento de flujo newtoniano $(n=1)$, de adelgazamiento por cizallamiento (pseudaplástico, $n<1)$ y de espesamiento por cizallamiento (dilatante, $n>1$ ). La consistencia y la fluencia se determinaron antes y después de la curva de empastamiento con el fin de determinar el efecto del procesamiento térmico sobre estos parámetros. Finalmente, los datos fueron suavizados empleando la función de Savistky-Golay en el programa GraphPad Prism versión 6.

\section{Perfil de viscosidad}

Las propiedades reológicas de cada dispersión se determinaron mediante un reómetro (TA INSTRUMENTS, AR 1500, New Castel, EE. UU.), equipado con una geometría de cilindro concéntrico. La velocidad de cizalla se mantuvo constante a $16,75 \mathrm{~s}^{-1}$, en todo el rango de calentamiento $\left(25^{\circ} \mathrm{C}-95^{\circ} \mathrm{C}-25^{\circ} \mathrm{C}\right)$ mientras que la velocidad de calentamiento fue de $10^{\circ} \mathrm{C} / \mathrm{min}$. El pico de viscosidad (PV), la caída de viscosidad (BD) y set back (SB) se registraron por duplicado (Pasqualone et al., 2021). Por último, mediante la función de Savistky-Golay se suavizaron los datos en el programa GraphPad Prism versión 6.

\section{Análisis estadístico}

Los efectos de la molienda abrasiva y el tratamiento térmico sobre las propiedades de flujo de las harinas de quinua (FP) y (PG) fue evaluado por medio del ANOVA. Para determinar las diferencias entre tratamientos se usó la prueba de Tukey, usando el programa Graphpad-6 (GraphPad Software, Inc., San Diego, CA, USA). En algunos casos, el valor-p fue calculado usando t-test para un análisis más profundo. 


\section{RESULTADOS Y DISCUSIÓN}

Todas las muestras utilizadas en este estudio se obtuvieron mediante molienda abrasiva del grano entero, obteniéndose una harina fina y un grano pulido, las cuales fueron sometidas a un proceso térmico; las condiciones experimentales se describen en la metodología.

\section{Propiedades de flujo}

La figura 1 muestra el comportamiento de la viscosidad antes (línea azul) y después (línea roja) del ciclo de calentamiento/enfriamiento a diferentes condiciones de estrés (esfuerzo de corte, $\mathrm{Pa}$ ) y velocidad de cizalla $\left(\mathrm{s}^{-1}\right)$. Además, muestra los coeficientes obtenidos por regresión del modelo de la potencia (Ecuación 1) donde (n) es el índice de flujo, (k) la consistencia y $\mathrm{R}^{2}$ el ajuste estadístico. Las dispersiones se sometieron a una variación en la velocidad de cizalla desde $0 \mathrm{~s}^{-1}$ a $300 \mathrm{~s}^{-1}$ y se observó el efecto de la velocidad de corte en la viscosidad. La figura 1a,b muestra el comportamiento de la viscosidad de la dispersión C-10-125-6\% (ver definición en tabla 1), antes del ciclo de calentamiento/enfriamiento, el comportamiento dilatante de la dispersión no se vió afectada por la velocidad de corte (puntos azules), esto se puede observar en los valores del índice n (1.563 y 1.437); lo anterior indica que la viscosidad de la dispersión aumenta con el incremento de la velocidad de corte (Gil-Poso, 2018). Un similar comportamiento ocurre con la dispersión C-40-125-6\% (figura 1c,d línea azul) antes del ciclo de calentamiento/enfriamiento.

Tabla 1: Descripción de las harinas de quinua y sus tratamientos

\begin{tabular}{|c|c|c|c|c|c|c|}
\hline Muestra & $\begin{array}{c}\text { Molienda } \\
\text { abrasiva (min) }\end{array}$ & $\begin{array}{c}\text { Granulometría } \\
(\mu \mathrm{m})\end{array}$ & Tratamiento térmico & $\begin{array}{l}\text { Índice de } \\
\text { flujo (n) }\end{array}$ & Consistencia (k) & $\begin{array}{c}\text { Ajuste } \\
R^{2}\end{array}$ \\
\hline C-10-125-6\% & 10 & 125 & N.A. & $1.44 \pm 0.08$ & $0.007 \pm 0.003$ & 0.9882 \\
\hline C-40-125-6\% & 40 & 125 & N.A. & $1.34 \pm 0.07$ & $0.012 \pm 0.004$ & 0.9911 \\
\hline C-10-125-12\% & 10 & 125 & N.A. & $1.20 \pm 0.04$ & $0.025 \pm 0.004$ & 0.9956 \\
\hline C-40-125-12\% & 40 & 125 & N.A. & $1.21 \pm 0.04$ & $0.024 \pm 0.005$ & 0.9941 \\
\hline FP-10-125-6\% & 10 & 125 & N.A. & $1.53 \pm 0.05$ & $0.006 \pm 0.001$ & 0.9966 \\
\hline FP-40-125-6\% & 40 & 125 & N.A. & $1.32 \pm 0.05$ & $0.013 \pm 0.003$ & 0.9950 \\
\hline FP-10-125-T-6\% & 10 & 125 & $\begin{array}{l}90^{\circ} \mathrm{C} / 40 \mathrm{~min}+ \\
\text { secado } 50^{\circ} \mathrm{C} \text { for } 16 \mathrm{~h} .\end{array}$ & $1.22 \pm 0.06$ & $0.023 \pm 0.007$ & 0.9891 \\
\hline FP-40-125-T-6\% & 40 & 125 & $\begin{array}{l}90^{\circ} \mathrm{C} / 40 \mathrm{~min}+ \\
\text { secado } 50^{\circ} \mathrm{C} \text { for } 16 \mathrm{~h} .\end{array}$ & $1.29 \pm 0.06$ & $0.016 \pm 0.004$ & 0.9934 \\
\hline FP-10-250-T-6\% & 10 & 250 & $\begin{array}{l}90^{\circ} \mathrm{C} / 40 \mathrm{~min}+ \\
\text { secado } 50^{\circ} \mathrm{C} \text { for } 16 \mathrm{~h} .\end{array}$ & $1.21 \pm 0.04$ & $0.022 \pm 0.005$ & 0.9943 \\
\hline FP-40-250-T-6\% & 40 & 250 & $\begin{array}{l}90^{\circ} \mathrm{C} / 40 \mathrm{~min}+ \\
\text { secado } 50^{\circ} \mathrm{C} \text { for } 16 \mathrm{~h} .\end{array}$ & $1.19 \pm 0.04$ & $0.02250 \pm 0.004$ & 0.9948 \\
\hline C1-125-T-6\% & NA & 125 & $\begin{array}{l}90^{\circ} \mathrm{C} / 40 \mathrm{~min}+ \\
\text { secado } 50^{\circ} \mathrm{C} \text { for } 16 \mathrm{~h} .\end{array}$ & $1.465 \pm 0.04$ & $0.00764 \pm 0.005$ & 0.9934 \\
\hline
\end{tabular}

Un salto en el comportamiento de la viscosidad entre $170 \mathrm{~s}^{-1}$ y $200 \mathrm{~s}^{-1}$ puede observarse en las figuras 1a,c (puntos rojos). El efecto de este salto se puede observar en el cambio del índice de flujo ( $n$ ) y la consistencia (k), por ejemplo, la dispersión C-10-125-6\% (figura 1a) mostró un comportamiento dilatante donde el valor del índice $\mathrm{n}$ fue de 1.303, es decir, que este tipo de dispersión aumenta su viscosidad debido al aumento de la velocidad de la cizalla $\left(0 \mathrm{~s}^{-1}\right.$ a $\left.300 \mathrm{~s}^{-1}\right)$. Sin embargo, esta misma dispersión sometida a velocidades entre $0 \mathrm{~s}^{-1} \mathrm{a}_{170 \mathrm{~s}^{-1}}$ mostraron un comportamiento newtoniano con un valor $\mathrm{n}$ de 1.005 (figura $1 \mathrm{~b}$ ). De la misma manera, la velocidad de corte afectó significativamente cuando las dispersiones fueron sometidas al ciclo de calentamiento/ enfriamiento (ver puntos rojos), por lo tanto, se puede concluir que la viscosidad de la dispersión es dependiente de la velocidad de cizalla, esto es importante no solo en la formulación del producto, sino también en la ingeniería de procesos, por ejemplo, cuando las dispersiones se mueven a través de tuberías a altas velocidades de flujo por efecto de las bombas, lo cual puede deteriorar la textura de la dispersión en el transporte de un tanque a otro (Rao, 2014). Por otro lado, el cambio de comportamiento reológico de dilatante a newtoniano no fue observado en las dispersiones C-40-125-6\% (ver figura 1c,d) esto puede deberse a la diferencia en la composición entre las dispersiones C-10 y C-40, ya que la dispersión C-40 es más rica en almidón que la dispersión C-10.

La figura 2 muestra el comportamiento de las dispersiones a concentraciones del $6 \%$ y $12 \%$, cuando son sometidas a diferentes velocidades de corte, en este caso el rango de velocidad de cizalla fue de $0 \mathrm{~s}^{-1}{\mathrm{a} 200 \mathrm{~s}^{-1}}^{-1}$ con el fin de evitar el efecto que tienen la alta velocidad de cizalla. La dispersión C-10-125-6\% (figura 2a) presentó mayor contenido de almidón y proteína que la muestra C-40-125-6\% (figura 2c), esto podría confirmarse por espectroscopia FTIR (Roa et al., 2020). Independientemente de la composición de las harinas, las dispersiones $(6 \% \mathrm{p} / \mathrm{v})$ mostraron comportamiento dilatante antes del calentamiento (puntos azules) con valores de índice de flujo (1.437 y 1.338) para las dispersiones C-10 y C-40, respectivamente. Sin embargo, luego que las dispersiones fueron sometidas al ciclo de calentamiento/enfriamiento, estas adquieren un comportamiento newtoniano, es decir, los índices de flujo $\mathrm{n}$ tienden a un valor de 1 (puntos rojos en la figura 2a,c), este comportamiento newtoniano sería favorable en el desarrollo de formulación alimenticias liquidas. En el caso hipotético de 
seleccionar una de las dispersiones, se recomienda las dispersiones C-10, ya que contienen un mayor porcentaje de proteína y fibra en comparación a las dispersiones C-40.
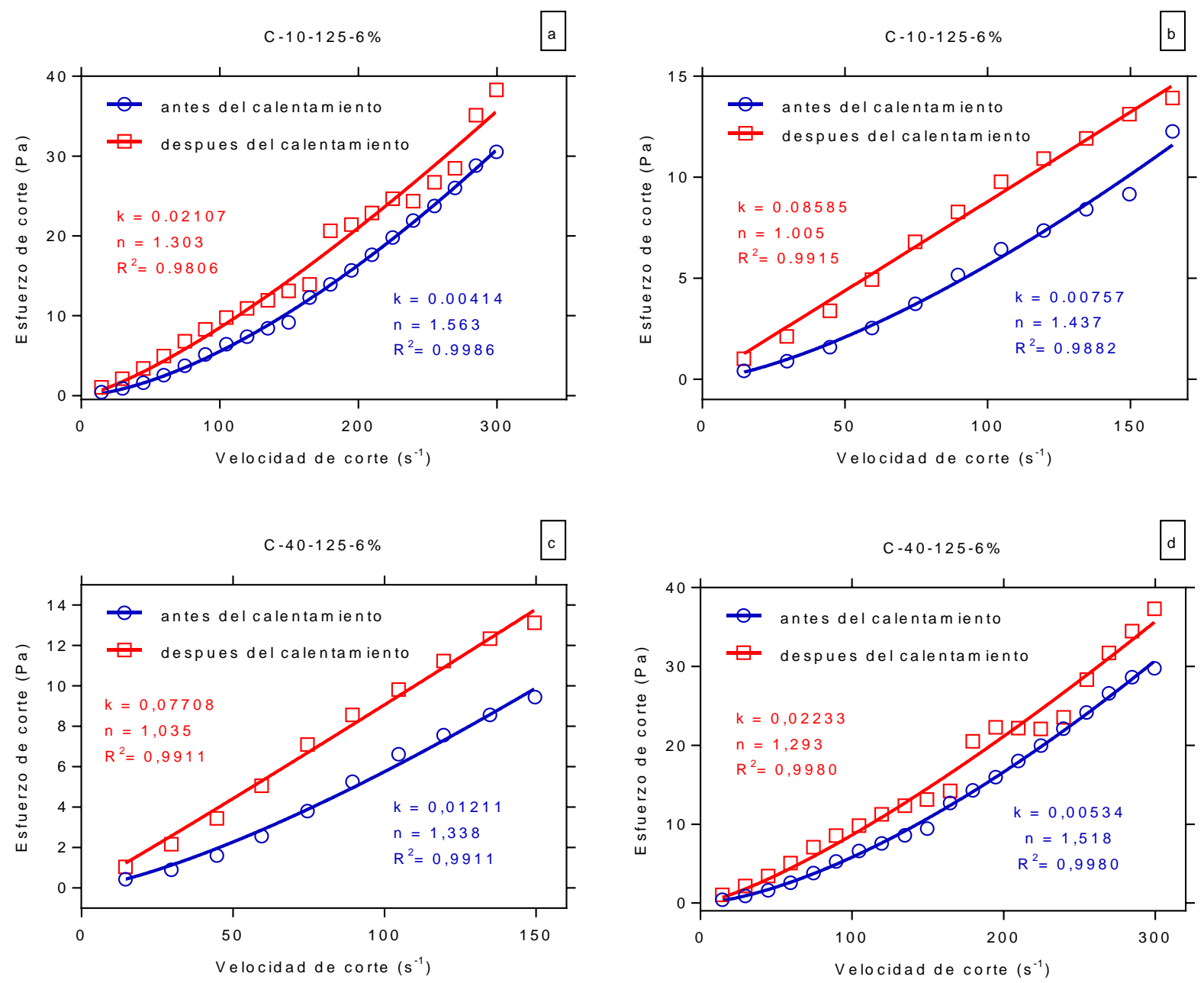

Fig. 1: Efecto de la velocidad de cizalla sobre el índice de flujo ( $n$ ) y la consistencia (k) de las dispersiones de harinas de grano pulido (C-10) y (C-40)

Si se compara el comportamiento reológico de las dispersiones al $6 \%$ y $12 \%$ en las figuras $2 a$,b se puede observar el cambio en los índices de flujo que evidencian una diferencia en el comportamiento reológico, pasando desde lo dilatante, newtoniano y pseudoplástico. Por ejemplo, el comportamiento de la viscosidad antes del calentamiento en las dos dispersiones siempre es dilatante con un índice de flujo $n>1$ (datos azules), Sin embargo, luego del calentamiento/enfriamiento la dispersión al 6\% (figura 2a) tiene comportamiento newtoniano $(\mathrm{n}=1.005)$, es decir su viscosidad no se ve afectada por el cambio en la velocidad de corte, lo que a la larga le puede dar más estabilidad en el tiempo. Por otra parte, la dispersión al 12\% (figura $2 \mathrm{~b}$ ) luego del calentamiento/enfriamiento se comporta pseudoplasticamente $(n=0.481)$, este comportamiento pseudoplástico puede deberse a que en el proceso de gelatinización los gránulos de almidón se rompen (Pereira et al., 2019), liberando amilosa al medio acuoso y luego en el enfriamiento estas cadenas de amilosa se alinean formado redes que hacen que se formen geles o suspensiones viscosas. Por lo tanto, entre más alta sea la concentración de la dispersión, más amilosa se libera al medio para forma un gel o suspensiones viscosas (Romano et al., 2020); sin embargo, esto no garantiza la estabilidad de estas suspensión cuando son sometidas a presiones generadas por la velocidad de cizallamiento

En la figura $2 \mathrm{~b}$ se observa el comportamiento pseudoplástico lo que quiere decir que la viscosidad disminuye con el aumento de la velocidad de corte, lo que podría ser un problema cuando el fluido es transportado en tuberías o cuando el producto es almacenado durante largo tiempos, su viscosidad final podría cambiar. Este cambio en el comportamiento reológico se debió al efecto de gelatinización sobre el gránulo de almidón y la desnaturalización de la proteína, lo que provoca el aumento de la viscosidad en la dispersión (esto se evidencia cuando se compara la viscosidad antes y después del ciclo de calentamiento/enfriamiento a una velocidad de corte constante). Para las dispersiones C-40 se observaron los mismos comportamientos. Arzuaga et al., (2021) observaron que las mezclas húmedas producidas en diferentes condiciones mostraron comportamientos de flujo variables luego de ser sometidas a tratamientos térmicos y de homogeneización. 

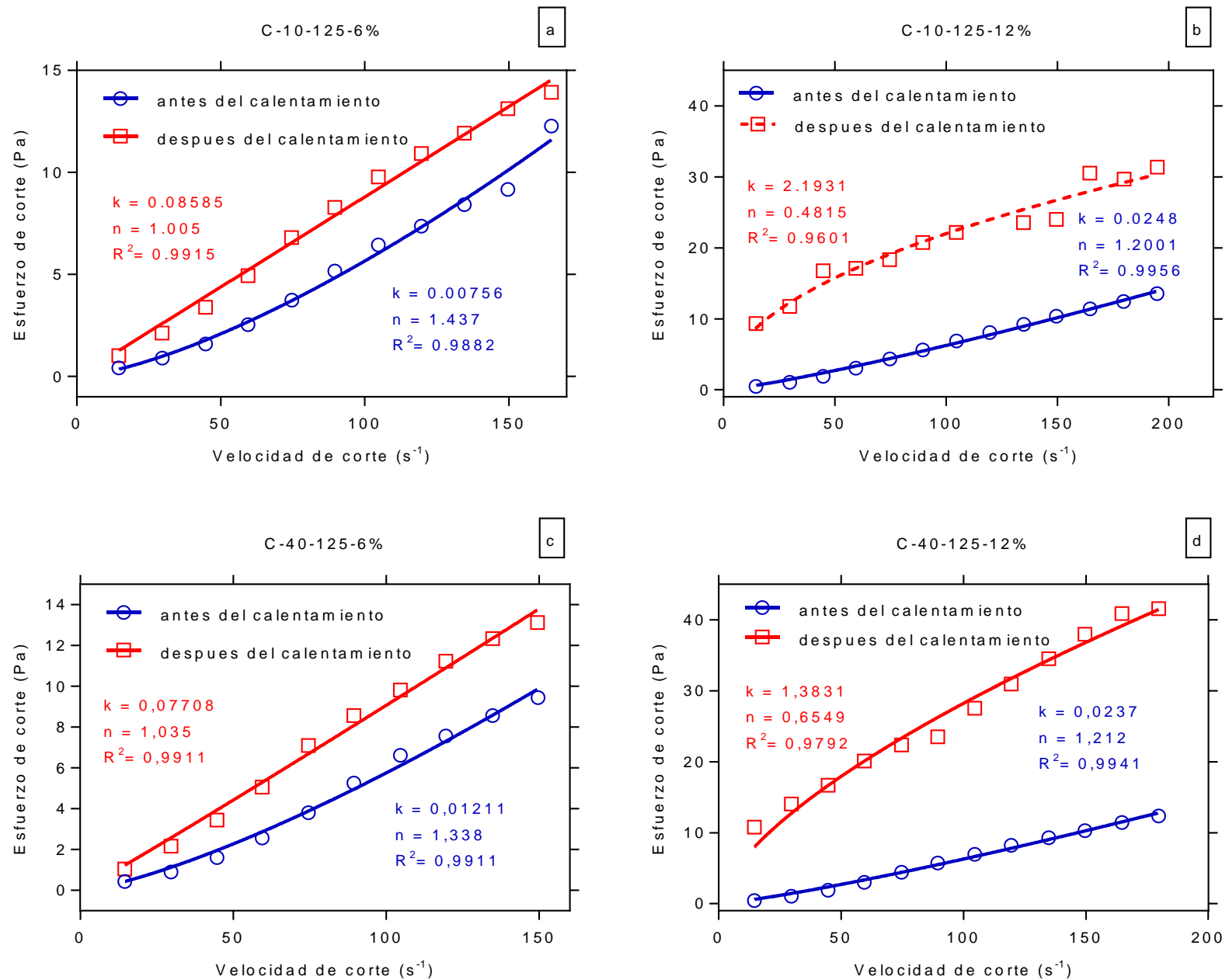

Fig. 2: Efecto de la velocidad de cizalla sobre el índice de flujo (n) y la consistencia (k) de las dispersiones de harinas de grano pulido (C-10) y (C-40) a diferentes concentraciones del $6 \%$ y $12 \%$

La figura 3 muestra el efecto de la velocidad de cizalla y ciclos de calentamiento/enfriamiento sobre las harinas obtenidas del germen del grano de quinua con y sin tratamiento térmico. Se observa que antes del calentamiento/enfriamiento las harinas FP 10 y FP 40 tiene un comportamiento reológico de tipo dilatante (datos azules) con índice de flujo $\mathrm{n}>1$ (figura 3a,c); estas harinas aunque tienen diferente composición siendo la FP40 rica en proteína y grasas no logran cambiar su comportamiento dilatante cuando es sometida a diferentes velocidad de cizalla. Por otra parte, este tipo de harinas de germen mostraron un comportamiento similar a las harinas de grano pulido, cuando fueron sometidas al calentamiento/enfriamiento, esto se evidencia en que cambian su comportamiento de dilatante a newtoniano.

Es importante destacar que se observa un salto entre el comportamiento de la viscosidad antes y después del calentamiento, este comportamiento es normal debido a la gelatinización del almidón, sin embargo, cuando las harinas del germen son sometidas al tratamiento térmico y luego secadas antes de la medición en el reómetro, este salto desaparece (figura $3 b, \mathrm{~d}$ ); otra diferencia importante es que las harinas de germen tratadas térmicamente no pierden su comportamiento dilatante cuando son sometidas al proceso de calentamiento/enfriamiento, este resultado podría emplearse en la formulación de bebidas instantáneas que cuando son mezcladas por acción mecánica, estas podrían ganar viscosidad y generar una bebida más espesa que el agua o por ejemplo, estas dispersiones no perderían viscosidad en procesos térmicos como la pasteurización. Por otro lado, si la harina de germen modificada térmicamente se emplea como harina instantánea, los índices de flujo (n) no cambian (datos azules), lo cual, nos muestran estabilidad en las dispersiones (ver datos azules) (figura 3c,d).

Arzuaga et al., (2021) observaron que después de la pasteurización, la curva de flujo mostró una tendencia a cambiar de newtoniana a tixotrópica. En la figura 4 se observa el comportamiento reológico de las dispersiones que fueron preparadas con harinas de diferentes granulometrías, no se observa grandes diferencias significativas entre las muestras entre el rango de $125 \mu \mathrm{m}$ y $250 \mu \mathrm{m}$, este resultado es contrario a lo observado por Bala et al. (2020) cuando determinaron el comportamiento reológico de la Almorta (Lathyrus sativus). 

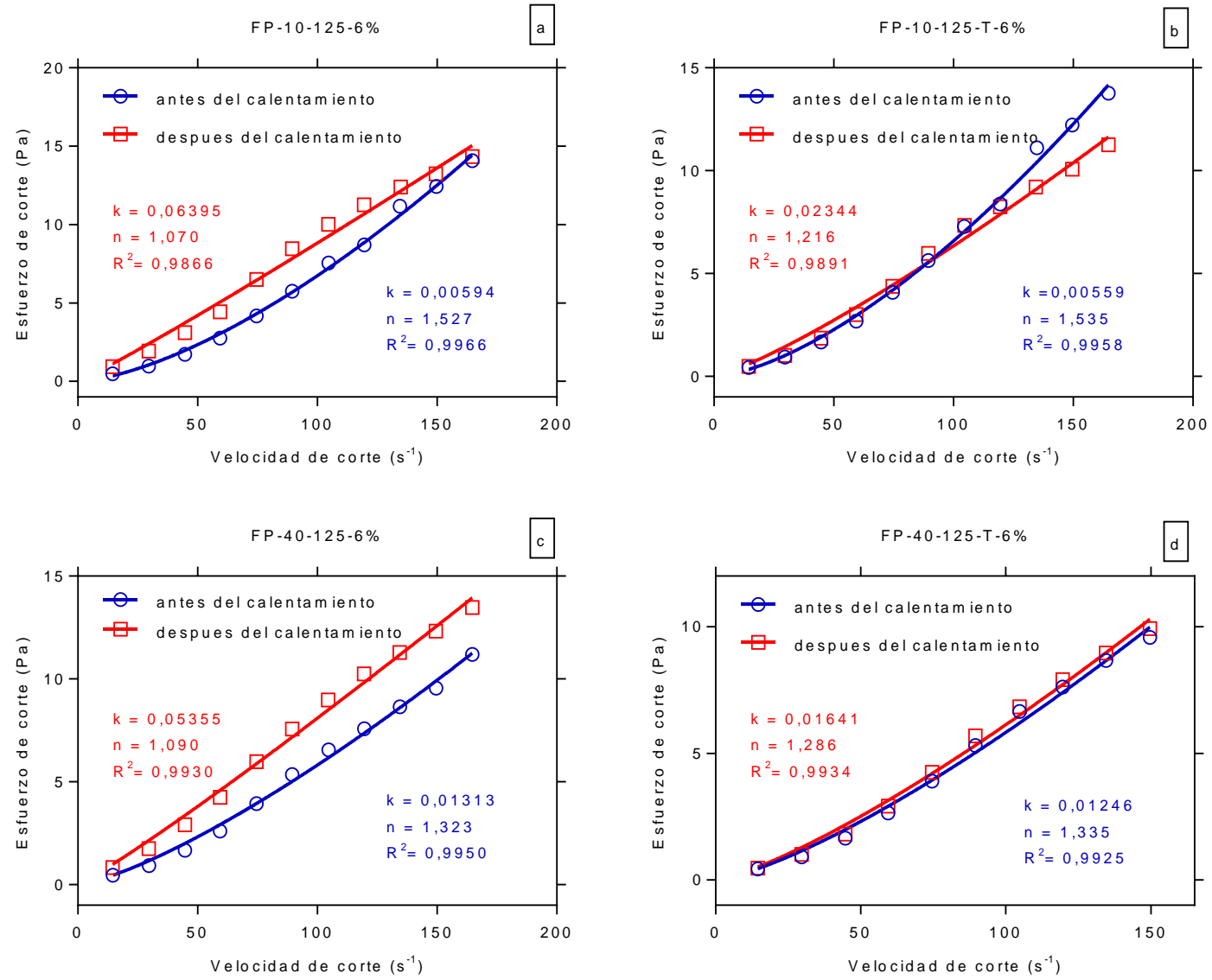

Fig. 3: Efecto de la velocidad de cizalla sobre el índice de flujo (n) y la consistencia (k) de las dispersiones de harinas del germen del grano (FP-10), (FP-40), (FP-10-T) y (FP-40-T)
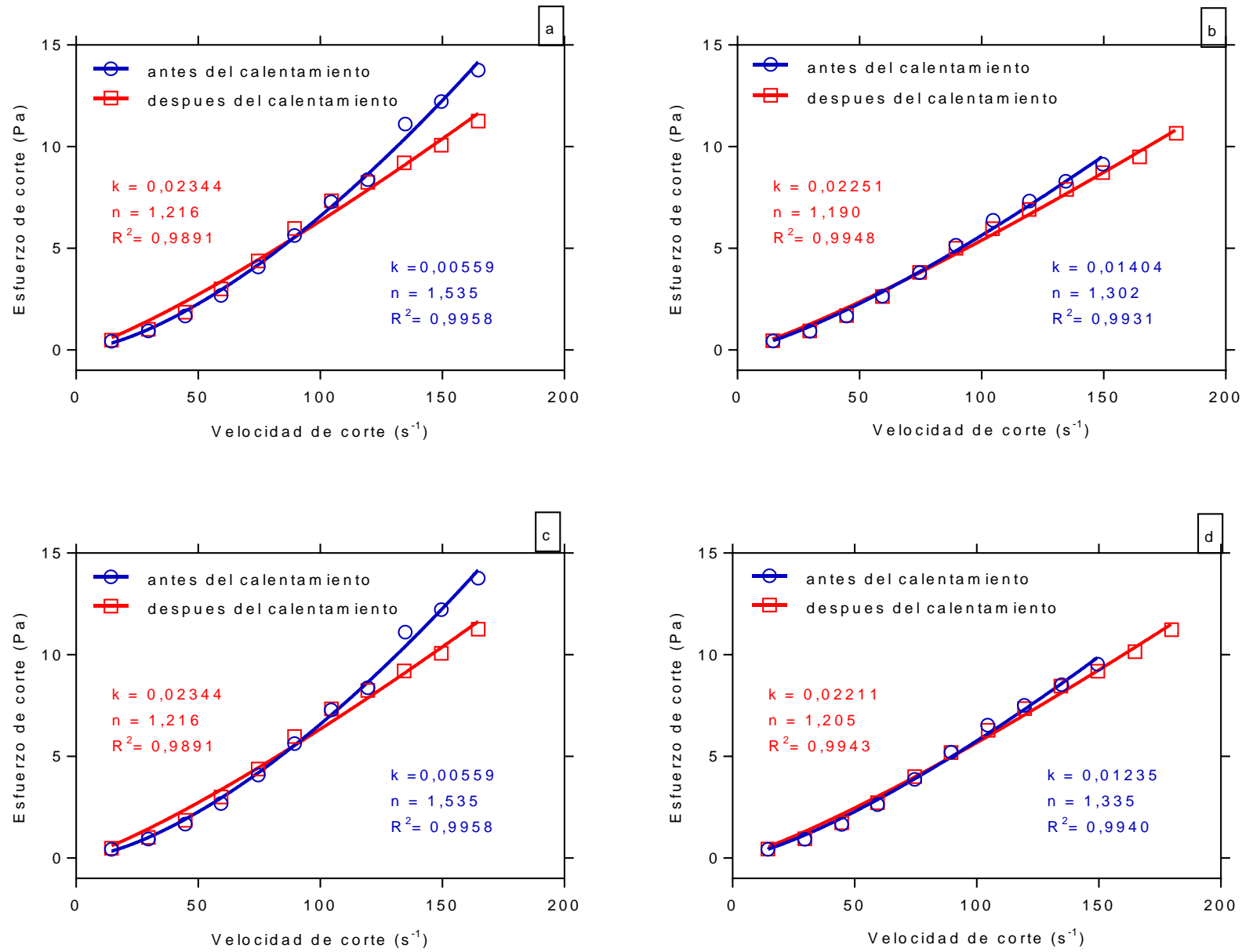

Fig. 4: Efecto de la velocidad de cizalla sobre las dispersiones preparadas con harinas de germen a diferentes granulometrías (125 micras y 250 micras) 
La estadística se realizó primero por medio del Anova, el resultado mostro un valor-p $<0.0001$ con un ajuste de $\mathrm{R}^{2}=0.98$ (Tabla 2). Esto que indica que existía por lo menos un valor del índice de flujo diferente, luego con el fin de determinar cuáles eran las muestras con índice de flujo diferentes y como podrían agrupan según el comportamiento de su flujo, se realizó la prueba de comparación múltiple tipo Tukey`s. La tabla 2 muestra los valores del índice de flujo (n) y los análisis estadísticos, los valores han sido agrupados en conjunto de letras, las muestras con letras semejantes indican comportamiento de flujo similar, mientras diferentes letras representan diferencias significativas en el valor del índice de flujo de cada dispersión, por ejemplo, hay 14 valores del índice de flujo $(n)$ agrupados en un rango de letras desde la $(a-I)$, siendo el valor alto (a) y el valor bajo (I).

\section{Perfil de viscosidad}

El perfil de viscosidad se realizó para determinar la evolución de la viscosidad durante la fase de calentamiento y enfriamiento, en las figuras $5 a, b, c$ se identifican dos zonas $Z 1$ y Z2, las cuales permiten identificar y explicar lo que sucede en las dispersiones durante el calentamiento. La figura 5 a muestra el comportamiento de la viscosidad de las muestras FP-10-125-6\%, mientras que la figura $5 b$ hace referencia a la muestra FP-40-125-6\% (línea azul continua); si se compara las formas de las curvas se observar las siguientes similitudes: En el perfil de viscosidad de la muestra FP-10-125-6\%, se observa un hombro a los 11.7 min y 65으, este mismo hombro se transforma en un pico definido en la muestra FP-40-125-6\%; este pico generalmente es atribuido a la gelatinización del almidón, proceso que ocurre a temperaturas entre los $60{ }^{\circ} \mathrm{C}$ hasta los $80^{\circ} \mathrm{C}$, aproximadamente (Zona Z-1). Mientras que en la muestra FP-40 (figura 5b) se logra observar un pico bien definido, en este mismo rango de temperatura.

Tabla 2: Análisis estadístico Anova y test de comparación múltiple de Tukey`s del valor del índice de flujo (n) de cada dispersión

\begin{tabular}{|c|c|c|c|}
\hline Tratamiento & Índice de flujo (n) & Tratamiento & Índice de flujo (n) \\
\hline C-10-125-6\% frío & $1.44 \pm 0.08^{\text {a.b.c }}$ & FP-10-125-6\% frío & $1.53 \pm 0.05^{\mathrm{a}, \mathrm{b}}$ \\
\hline C-10-125-6\%-caliente & $1.01 \pm 0.04^{h, i, j}$ & FP-10-125-6\% caliente & $1.07 \pm 0.06^{f, g, h, i}$ \\
\hline C-10-125-12\%-frío & $1.21 \pm 0.04^{\mathrm{d}, \mathrm{e}, \mathrm{f}, \mathrm{g}, \mathrm{h}}$ & FP-10-125-12\% frío & $0.91 \pm 0.02^{i, j}$ \\
\hline C-10-125-12\%-caliente & $0.48 \pm 0.04^{1}$ & FP-10-125-12\% caliente & $0.84 \pm 0.03^{\mathrm{j}, \mathrm{k}}$ \\
\hline C-40-125-6\%-frío & $1.34 \pm 0.07^{a, b, c, d}$ & FP-10-125-T-6\% frío & $1.54 \pm 0.05^{a}$ \\
\hline C-40-125-6\%-caliente & $1.04 \pm 0.05^{g, h, i, j}$ & FP-10-125-T-6\% caliente & $1.22 \pm 0.06^{\mathrm{d}, \mathrm{e}, \mathrm{f}, \mathrm{g}}$ \\
\hline C-40-125-12\%-frío & $1.21 \pm 0.04^{\mathrm{d}, \mathrm{e}, \mathrm{f}, \mathrm{g}}$ & FP-10-250-T-6\% frío & $1.34 \pm 0.05^{a, b, c, d}$ \\
\hline C-40-125-12\%-caliente & $0.66 \pm 0.04^{\mathrm{k}, \mathrm{I}}$ & FP-10-250-T-6\% caliente & $1.21 \pm 0.04^{\mathrm{d}, \mathrm{e}, \mathrm{f}, \mathrm{g}}$ \\
\hline C-250-T-6\%-frío & $1.44 \pm 0.08^{\mathrm{a}, \mathrm{b}, \mathrm{c}}$ & FP-40-125-6\% frío & $1.32 \pm 0.05^{a, b, c, d}$ \\
\hline C-250-T-6\%-caliente & $1.23 \pm 0.03^{\mathrm{c}, \mathrm{d}, \mathrm{e}, \mathrm{f}, \mathrm{g}}$ & FP-40-125-6\% caliente & $1.09 \pm 0.04^{e, f, g, h, i}$ \\
\hline C1-125-T-6\%-frío & $1.31 \pm 0.07^{\mathrm{c}, \mathrm{d}, \mathrm{e}}$ & FP-40-125-T-6\% frío & $1.34 \pm 0.06^{\mathrm{a}, \mathrm{b}, \mathrm{c}, \mathrm{d}}$ \\
\hline C1-125-T-6\%-caliente & $1.17 \pm 0.05^{\mathrm{d}, \mathrm{e}, \mathrm{f}, \mathrm{g}, \mathrm{h}}$ & FP-40-125-T-6\% caliente & $1.29 \pm 0.06^{\mathrm{c}, \mathrm{d}, \mathrm{e}, \mathrm{f}}$ \\
\hline C2-125-T-6\%-frío & $1.30 \pm 0.06^{\mathrm{c}, \mathrm{d}, \mathrm{e}}$ & FP-40-250-T-6\% frío & $1.31 \pm 0.06^{\mathrm{b}, \mathrm{c}, \mathrm{d}, \mathrm{e}}$ \\
\hline C2-125-T-6\% caliente & $1.18 \pm 0.04^{\mathrm{d}, \mathrm{e}, \mathrm{f}, \mathrm{g}, \mathrm{h}}$ & FP-40-250-T-6\% caliente & $1.19 \pm 0.04^{\mathrm{d}, \mathrm{e}, \mathrm{f}, \mathrm{g}, \mathrm{h}}$ \\
\hline
\end{tabular}
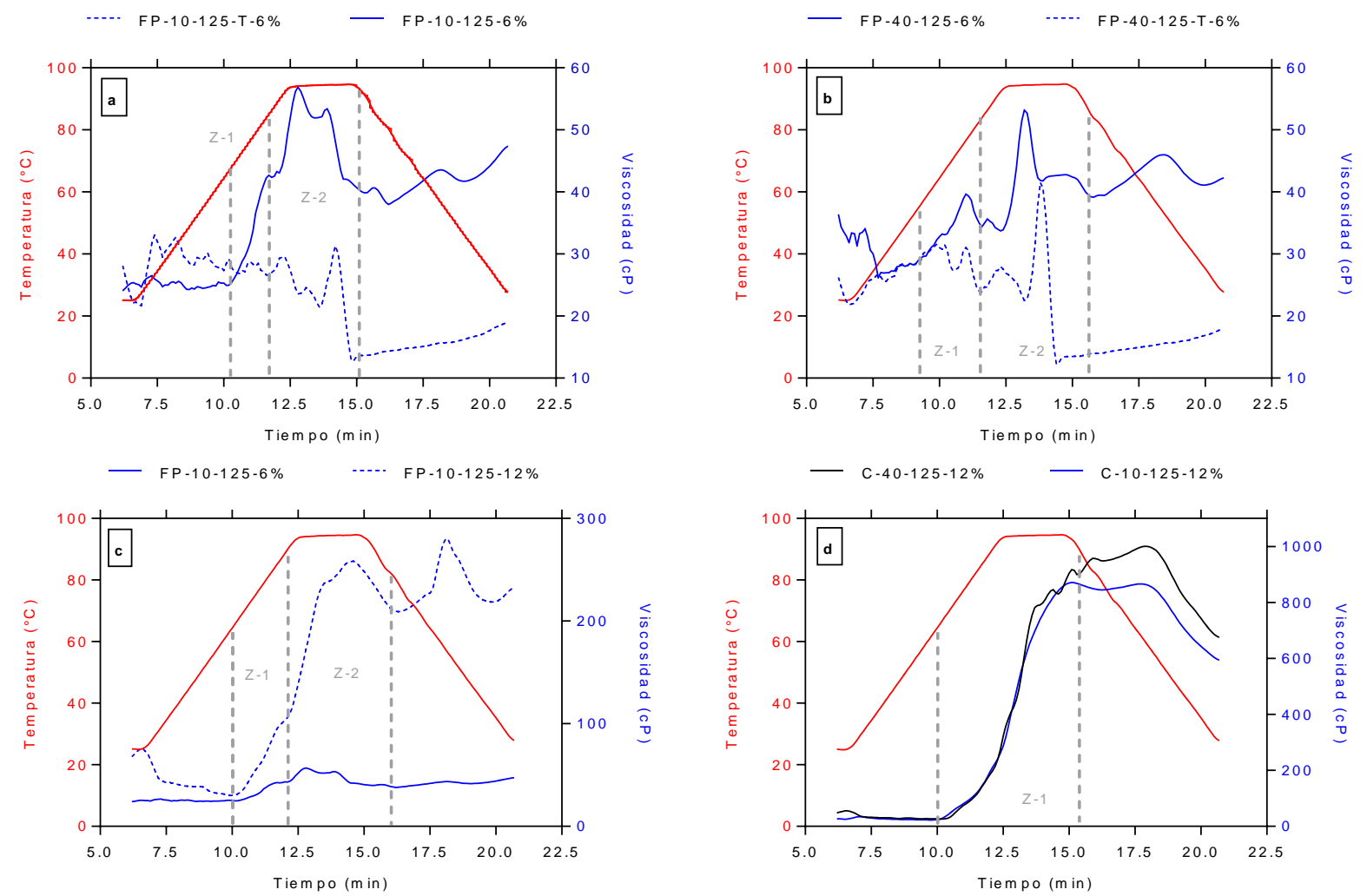

Fig. 5: perfil de viscosidad de las dispersiones sometidas a un ciclo de calentamiento y enfriamiento $\left(25^{\circ} \mathrm{C}-95^{\circ} \mathrm{C}-25^{\circ} \mathrm{C}\right)$ 
Una posible explicación al porque en la muestra FP-10 solo se observa un hombro en lugar de un pico, se debe precisamente al pulimiento por capas del grano durante la molienda abrasiva por 10 minutos, lo que con lleva a que se obtenga gránulos de almidón ubicados en la parte superficial del perisperma, mientras que en la muestra FP-40 los gránulos de almidón se presentan en mayor cantidad, ya que el proceso abrasivo es más intenso (40 minutos) logrando desprender más partes del perisperma almidonoso. Bala et al., (2020) encontraron diferencias composicionales en las harinas de sorgo cuando fueron sometidas al proceso de molienda abrasiva, este tipo de harinas tenía comportamientos reológicos diferentes, ya que su composición variaba de acuerdo con la granulometría y/o al tiempo de operación. Bajo estas condiciones de procesamiento, el almidón aporta $40 \mathrm{cP}$ en la viscosidad de la dispersión.

El pico máximo de viscosidad para las muestras FP-10 y FP-40 ocurre entre los 11.5 y 15 minutos, ósea durante la fase de calentamiento (zona Z-2) en un rango de temperaturas de $83^{\circ} \mathrm{C}$ a $95^{\circ} \mathrm{C}$. Durante esta fase ocurre un incremento de la viscosidad desde $43 \mathrm{cP}$ hasta $56 \mathrm{cP}$ para la dispersión FP-10, mientras que, para la dispersión FP-40 la viscosidad aumenta desde $35 \mathrm{cP}$ hasta $54 \mathrm{cP}$, aproximadamente. Este incremento en la viscosidad puede ser atribuido a la desnaturalización y desdoblamiento de la proteína presente, la cual puede formar redes tipo gel con el agua y con los fragmentos de amilosa que son liberados luego de la gelatinización del almidón; posiblemente esta sea una de las razones, por la cual, el incremento de la viscosidad sea mayor en la muestra FP-40, ya que esta muestra tiene un contenido de proteína de aproximadamente el $35 \%$ y que es mayor al contenido de proteína de la muestra FP-10. Romero y Zhang (2019) observaron este comportamiento cuando estudiaron cuatro variedades de frijol en la elaboración de pastas libre de gluten.

En estudios anteriores sobre las propiedades térmicas del aislado de quinua. El-Sohaimy et al. (2018) y Roa et al. (2019) lograron identificar que estas temperaturas están asociadas a la desnaturalización de diferentes tipos de proteínas presentes en la quinua, esto brinda las bases para poder afirmar lo anterior. Se observa una caída de la viscosidad hasta los $50 \mathrm{cP}$ durante la etapa de calentamiento constante $\left(95^{\circ} \mathrm{C}\right)$ y posteriormente hay una leve recuperación de la viscosidad durante la etapa de enfriamiento, esta recuperación de la viscosidad está asociada a los procesos de retrogradación de la amilosa, fenómeno que ha sido ampliamente discutido en la literatura (El-Sohaimy et al., 2015; Romero y Zhang, 2019; Bala et al., 2020).

Las figuras también muestran el comportamiento de las harinas que fueron sometidas al tratamiento hidrotérmico a una temperatura de $90{ }^{\circ} \mathrm{C}$. Se observa que el hombro y el pico relacionado a la gelatinización del almidón desaparece (antes de los $12.5 \mathrm{~min}$ ) y solo se logran observar pequeños picos que pueden corresponder a pequeños fragmentos de gránulos de almidón que no gelatinizaron durante el tratamiento hidrotérmico, sin embargo, el pico que se observa después de los 12.5 minutos corresponde a la fracción proteica, esto es posible ya que la proteína de quinua inicia su proceso de desnaturalización después de los $80{ }^{\circ} \mathrm{C}$ (Mir et al., 2021) y el proceso hidrotérmico solo se realizó hasta los $90 \stackrel{\circ}{\circ}$, lo que permite que el proceso de desnaturalización de la proteína sea parcial y no total, conservando proteína nativa (Gajardo, 2005).

Es importante destacar que en la muestra FP-40 el pico asociado a la proteína es más intenso debido a que esta muestra contiene más proteína que la muestra FP-10. La figura $5 \mathrm{c}$ muestra el perfil de la viscosidad de las muestras FP-10 a concentraciones del $6 \% \mathrm{p} / \mathrm{v}$ y $12 \% \mathrm{p} / \mathrm{v}$, se observa que el aumento en la concentración causa un incremento de hasta $250 \mathrm{cP}$ en la viscosidad de la dispersión en la fase de calentamiento la cual ocurre hasta los 15 min del tratamiento, luego durante la fase de enfriamiento (18 minutos) se alcanza el pico de máxima viscosidad, esto es probable por el reacomodo y la interacción de las cadenas de fibras-proteínas y/o amilosaproteína (Villarroel et al., 2018). En la figura $5 \mathrm{~d}$ el perfil de la viscosidad es muy similar en las dos muestras, esto quiere decir que el efecto de la concentración al $12 \%$ es mayor que el efecto de la diferencia composicional entre las muestras, esta concentración del $12 \%$ genera altas viscosidades no apropiadas para formar o desarrollar bebidas liquidas de alto flujo.

\section{CONCLUSIONES}

De acuerdo a los resultados de este estudio y de su discusión, de la comparación con resultados de otros autores y del detallado análisis presentado se pueden extraer las siguientes conclusiones: 1) el modelo de la Potencia ajusto satisfactoriamente logrando determinar el índice de flujo de las dispersiones y de esta manera describir su comportamiento reológico; 2) las dispersiones a base de harina de germen obtenidas a 10 minutos de molienda mostraron un comportamiento dilatante mayor a las dispersiones a base de harina obtenidas a 40 minutos, sin embargo, después de tratamiento térmico las dispersiones se comportan como fluidos newtonianos 3 ) las dispersiones formuladas a partir del perisperma almidonoso tienen un comportamiento pseudoplástico en lugar de dilatante como las dispersiones a base de harina de germen; 4) el proceso térmico logra gelatinizar el almidón sin embargo no es capaz de desnaturalizar completamente la proteína de la quinua, logrando que estas harinas procesadas térmicamente adquieran propiedades tecnofuncionales en el desarrollo de nuevas formulaciones lo cual se evidencia en la estabilidad de la viscosidad frente a I ciclo de calentamiento y enfriamiento. 


\section{REFERENCIAS}

Arzuaga, M., Aalaei K., y otros cinco autores, Infant milk formulae processing: effect of wet-mix total solids and heat treatment temperature on rheological, emulsifying and nutritional properties, https://doi.org/10.1016/j.jfoodeng.2020.110194, J. Food Eng, 290, 9 (2021)

Asher, A., Galili S., Whitney T., y Rubinovich L., The potential of quinoa (Chenopodium quinoa) cultivation in Israel as a dualpurpose crop for grain production and livestock feed, https://doi.org/10.1016/j.scienta.2020.109534, Sci. Hortic., 272, (2020)

Awika, J., McDonough C., y Rooney L., Decorticating sorghum to concentrate healthy phytochemicals, https://doi.org/10.1021/jf0510384, J. Agric. Food Chem., 53(16), 6230-6234 (2005)

Bala, M., Handa S., Mridula D., y Singh R., Physicochemical, functional and rheological properties of grass pea (Lathyrus sativus L.) flour as influenced by particle size, https://doi.org/10.1016/j.heliyon.2020.e05471, Heliyon, 6(11) (2020)

Delgado, A., Palacios J., y Betancourt C., Evaluación de 16 genotipos de quinua dulce (Chenopodium quinoa Willd) en el municipio de lles, Nariño (Colombia), http://www.scielo.org.co/, Agronomía Colombiana, 27(2), 159-167 (2009)

Dussán-Sarria, S., De la Cruz-Noguera R., y Godoy S., Estudio del perfil de aminoácidos y análisis proximal de pastas secas extruidas a base de harina de quinua y harina de chontaduro, https://doi.org/10.4067/s0718-07642019000600093, Información Tecnológica, 30(6), 93-100 (2019)

El-Sohaimy, S., Mohamed S., y otros tres autores, Compositional analysis and functional characteristics of quinoa flour, https://doi.org/10.9734/ARRB/2018/38435, Annu. Res. Rev. Biol., 22(1), 1-11 (2018)

El-sohaimy, S., Refaay T., y Zaytoun M., Physicochemical and functional properties of quinoa protein isolate, https://doi.org/10.1016/j.aoas.2015.10.007, Ann. Agric. Sci., 60(2), 297-305 (2015)

Gajardo, I., Caracterización y determinación de la estabilidad durante el almacenamiento de las proteínas de harina de quinua orgánica sin pulir y pulida proveniente de la VI región de Chile, trabajo de grado ingeniero en alimentos, Departamento de Ciencia de Los Alimentos y Tecnología Química, Universidad de Chile (2005)

Gil-Poso, J., Evaluación de modificadores reológicos en una formulación de shampoo como alternative al uso del cloruro de Sodio, Trabajo de grado en modalidad de investigación, maestría en ciencias y tecnologías cosméticas, Universidad Politécnica Salesiana (2018)

Gupta, A., Sharma S., y Reddy V., Quinoa protein isolate supplemented pasta: nutritional, physical, textural and morphological characterization, https://doi.org/10.1016/j.Iwt.2020.110045, LWT- Food Sci. Technol., 135, 7 (2021)

Mir, N., Riar C., y Singh S., Improvement in the functional properties of quinoa (Chenopodium quinoa) protein isolates after the application of controlled heat-treatment: effect on structural properties, https://doi.org/10.1016/j.foostr.2021.100189, Food Structure, 28 (2021)

Mulcahy, E., Fargier-Lagrange M., Mulvihill D., y O'Mahony J., Characterization of heat-induced protein aggregation in whey protein isolate and the influence of aggregation on the availability of amino groups as measured by the ortho-

phthaldialdehyde (OPA) and trinitrobenzenesulfonic acid (TNBS) methods, https://doi.org/10.1016/j.foodchem.2017.01.155, Food Chem., 229, 66-74 (2017)

Pasqualone, A., Constantini M., y otros dos autores, Production of extruded-cooked lentil flours at industrial level: effect of processing conditions on starch gelatinization, dough rheological properties and techno-functional parameters, https://doi.org/10.1016/j.Iwt.2021.111580, LWT, 147 (2021)

Pereira, E., Encina-Zelada C., y otros cuatro autores, Chemical and nutritional characterization of Chenopodium quinoa Willd (quinoa) grains: a good alternative to nutritious food, https://doi.org/10.1016/j.foodchem.2018.12.068, Food Chem., 280, 110$114(2019)$

Rao, M., Rheology of fluid, semisolid, and solid foods principles and applications. 3 ${ }^{\mathrm{a}}$ Ed., 418-422. Springer New York (2014)

Roa, D., Bravo J., y otros tres autores, Hyper-protein quinoa flour (Chenopodium Quinoa Wild): monitoring and study of structural and rheological properties, https://doi.org/10.1016/j.Iwt.2019.108952, LWT- Food Sci. Technol., 121, 32 (2019)

Roa, D., Solanilla J., y otros tres autores, Structural and thermal properties of the amaranth starch granule obtained by highimpact wet milling, https://doi.org/10.1515/ijfe-2020-0024, Int. J. Food Eng., 16(10), 12 (2020)

Romano, N., Ureta M., Guerrero M., y Gómez A., Nutritional and technological properties of a quinoa (Chenopodium quinoa Willd.) spray-dried powdered extract, https://doi.org/10.1016/j.foodres.2019.108884, Int. Food Res. J, 129, 41 (2020)

Romero, H., y Zhang Y., Physicochemical properties and rheological behavior of flours and starches from four bean varieties for gluten-free pasta formulation, https://doi.org/10.1016/j.jafr.2019.100001, J. Agric. Food Res., 1, 8 (2019)

Song, J., Yan Y., y otros cinco autores, Characterization of fatty acids, amino acids and organic acids in three colored quinoas based on untargeted and targeted metabolomics, https://doi.org/10.1016/j.Iwt.2020.110690, LWT- Food Sci. Technol., 140, 31 (2021)

Van de Vondel, J., Lambrecht M., y otros cuatro autores, Impact of hydrothermal treatment on denaturation and aggregation of water-extractable quinoa (Chenopodium quinoa Willd.) protein, https://doi.org/10.1016/j.foodhyd.2021.106611, Food Hydrocolloids, 115 (2021) 
Villarroel, P., Gómez C., y otros dos autores, Almidón resistente: características tecnológicas e intereses fisiológicos, Revista Chilena de Nutrición, 45(3), 271-278 (2018)

Warncke, M., y Kulozik U., Impact of temperature and high-pressure homogenization on the solubility and rheological behavior of reconstituted dairy powders of different composition, https://doi.org/10.1016/j.powtec.2020.08.039, Powder Technol., 376, 285-295 (2020) 
